\title{
NOVEL DIAPHRAGM BASED STIRLING CRYOCOOLER
}

\author{
A.J. Caughley ${ }^{1}$, A Tucker ${ }^{2}$, M Gschwendtner ${ }^{3}$, and M Sellier ${ }^{2}$ \\ ${ }^{1}$ Industrial Research Ltd, Christchurch, New Zealand \\ ${ }^{2}$ University of Canterbury, Christchurch, New Zealand \\ ${ }^{3}$ TS-dot Engineering, Christchurch, New Zealand
}

\begin{abstract}
Industrial Research Ltd has developed a unique diaphragm-based pressure wave generator technology for employment in pulse tube and Stirling cryocoolers. The system uses a pair of metal diaphragms to separate the clean cryocooler gas circuit from a conventionally lubricated mechanical driver, thus producing a clean pressure wave with a long-life drive. We have now extended the same diaphragm concept to support and seal the displacer in a free piston Stirling expander. The diaphragms allow displacer movement without rubbing or clearance gap seals, hence allowing for the development of costeffective long-life and efficient Stirling cryocoolers. Initial modeling, operating in conjunction with a 200 cc swept volume pressure wave generator, predicted in excess of $300 \mathrm{~W}$ cooling at $77 \mathrm{~K}$ with a Carnot efficiency of over 25\%. A proof-of-concept prototype has achieved cryogenic temperatures. Details of the concept, modeling, and testing will be presented.
\end{abstract}

KEYWORDS: Stirling, cryocooler, diaphragm, free piston.

\section{INTRODUCTION}

Metallic diaphragm pressure wave generators (PWG) developed by Industrial Research Ltd [1] have matured to provide a useful alternative to linear motor pressure wave generators for Stirling and pulse tube cryocoolers. The metallic diaphragm pressure wave generator employs two opposed diaphragms that balance each other's average gas forces. A conventionally lubricated, ambient pressure mechanism sits between the two diaphragms to reciprocate the diaphragms. This paper describes adaptation of the diaphragm system to a free piston Stirling cryocooler. 


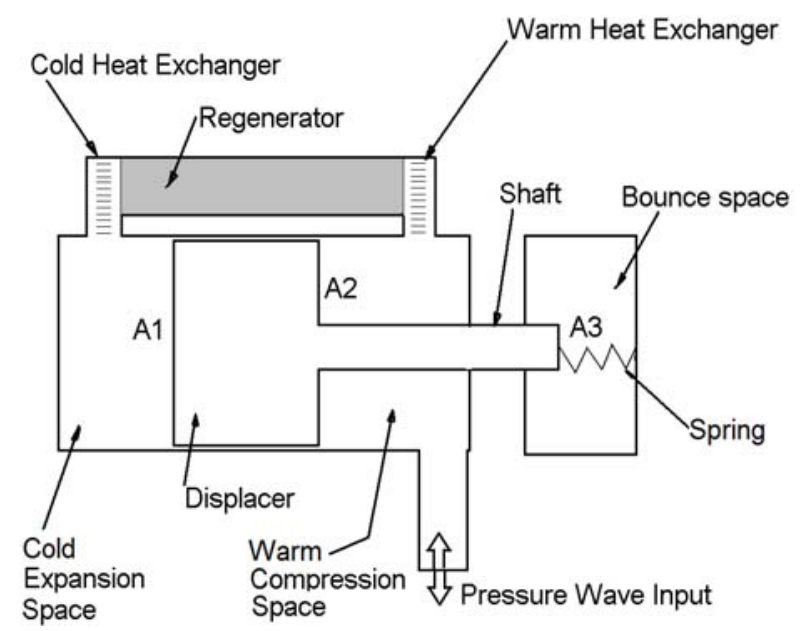

FIGURE 1. Schematic diagram of a typical free piston cold head.

Beale [2] and Cooke-Yarborough [3] were early pioneers of free piston Stirling machines. Their focus was Stirling engines for remote power applications. Beale used conventional pistons and his machines saw commercial success through companies such as Sunpower [4]. Cooke-Yarborough's invention employed a diaphragm, and, though not commercialized, his most successful prototype ran continuously for 12 years [5].

FIGURE 1 is a schematic of a conventional free piston Stirling cryocooler. A pressure wave is supplied to the cold head via a duct. A displacer reciprocates the working gas between the cold expansion and the warm compression spaces. As the gas moves from the warm to cold end, it passes through a regenerator transferring its heat to the regenerator's matrix and regains the heat on return, thus maintaining a large temperature gradient. The displacer movement is timed in such a way that the majority of the gas is in the warm end for the compression part of the pressure cycle and in the cold end for the expansion part. Instead of using a mechanically driven displacer as seen in the Phillips Stirling [6] cryocoolers, the free piston Stirling system uses differential gas pressure on the ends of the displacer for movement. The warm side of the displacer is divided into two regions: one region experiences the supplied pressure wave (A2), and the other region (A3) is kept near the average system pressure. In conventional free piston systems the displacer's guiding shaft goes through a clearance seal into a chamber called the bounce space. The relative areas A1, A2, A3 are chosen to provide the required amplitude and phase of the driving force. Displacer drift is controlled by a small spring. As with all cryocoolers, cleanliness of the working gas is important. Volatile components from lubricants in the working gas can collect and block the regenerator; likewise, if the displacer rubs against its walls wear, particles will collect in the regenerator. Precise radial location of the displacer is normally achieved by use of gas or flexure bearings; gas bearings have limited capacity and are therefore best for small cryocoolers whereas flexure bearings require precise alignment during assembly, which increases cost. Clearance gap seals have leakage, which produces a performance loss. This paper investigates the concept of using metallic diaphragms to seal and support the displacer in a free piston Stirling cold head. Diaphragms have the potential to support a large displacer without appendix gap leakage; they are tolerant of alignment errors; and their short stroke minimizes vibration. 


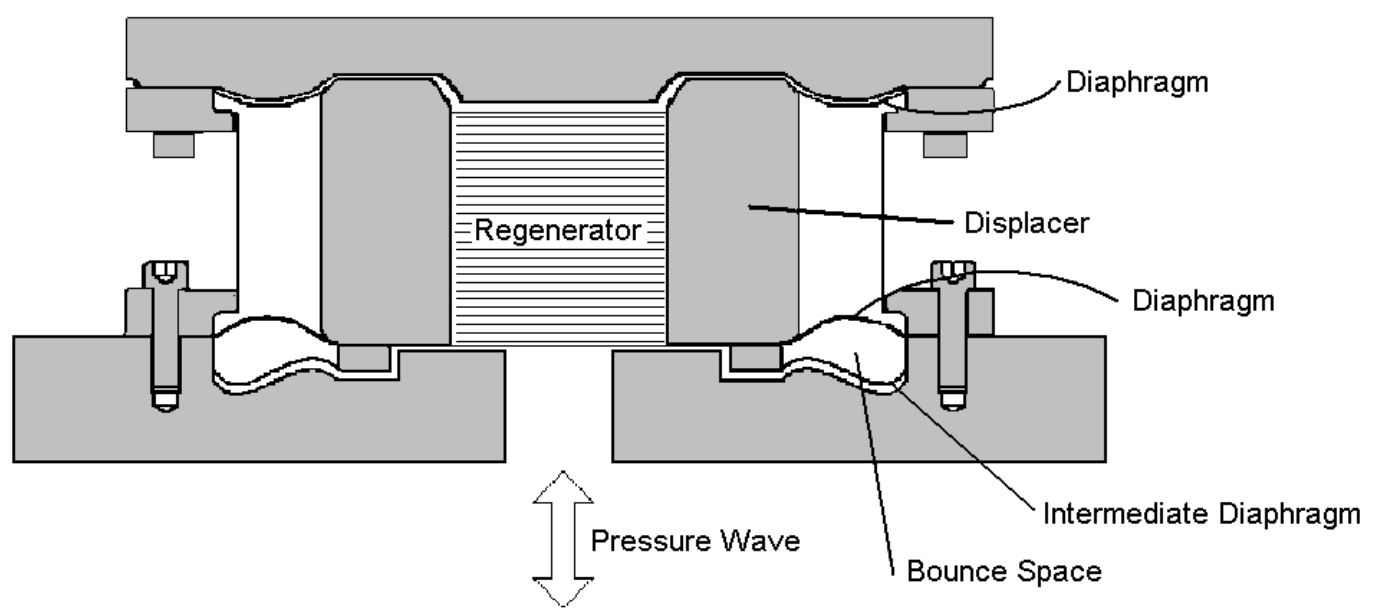

FIGURE 2. Conceptual layout of a diaphragm free piston Stirling cold head

\section{A FREE PISTON STIRLING EXPANDER WITH DIAPHRAGMS}

The metallic diaphragm PWG's concept has the potential to produce a free piston Stirling cold head without rubbing or high precision clearance seal parts. FIGURE 2 shows the concept explored. Two diaphragms were used to seal and suspend the displacer. As with the PWG, the diaphragms seal in the cryocooler's working gas. Equal sized diaphragms balance the large compressive forces on the displacer, leaving the displacer free to move. The stiffness of the diaphragms provides a spring to centre the displacer. The diaphragms' high radial stiffness limits the displacer's movement to the axial direction. The diaphragms' large area achieves the required swept volume with a small linear motion. The regenerator is housed inside the displacer thereby adding to its mass thus lowering its resonant frequency and improving stroke and phase angle. The displacer can be pneumatically actuated as per a conventional free piston machine by dividing its warm end into two regions: one that experiences the pressure wave, and one that remains at the average pressure. The area division is provided by a third diaphragm separating an outer annular ring for a bounce space. A slow leak to the bounce space ensures it remains at the average system pressure. Alternatively a central 'shaft' can be used to provide a piston/dashpot effect. The end result is a displacer with no rubbing parts and no fine tolerance clearance gap seals. The large surface areas and radial flows of the diaphragms offered the opportunity for heat transfer without expensive heat exchangers. This possibility was indicated previously by Caughley et al [1] who successfully ran a pulse tube with diaphragm PWG using the diaphragm as the after-cooler. In this case the PWG area plus the transfer holes to the cold head's compression space provided the after cooler. The cold heat exchanger relied solely on the expansion diaphragm's large area.

\section{MODELLING AND PREDICTIONS}

A numerical model of the diaphragm cold head concept was developed with industrystandard software Sage [7]. Sage is a one-dimensional frequency domain modeler for oscillating thermodynamic systems such as Stirling engines and refrigerators. Sage's onedimensional idealization does not take into account the radial flow effects, which contribute to heat transfer in the diaphragm compression and expansion spaces so should provide a conservative estimate of heat transfer in those components. 


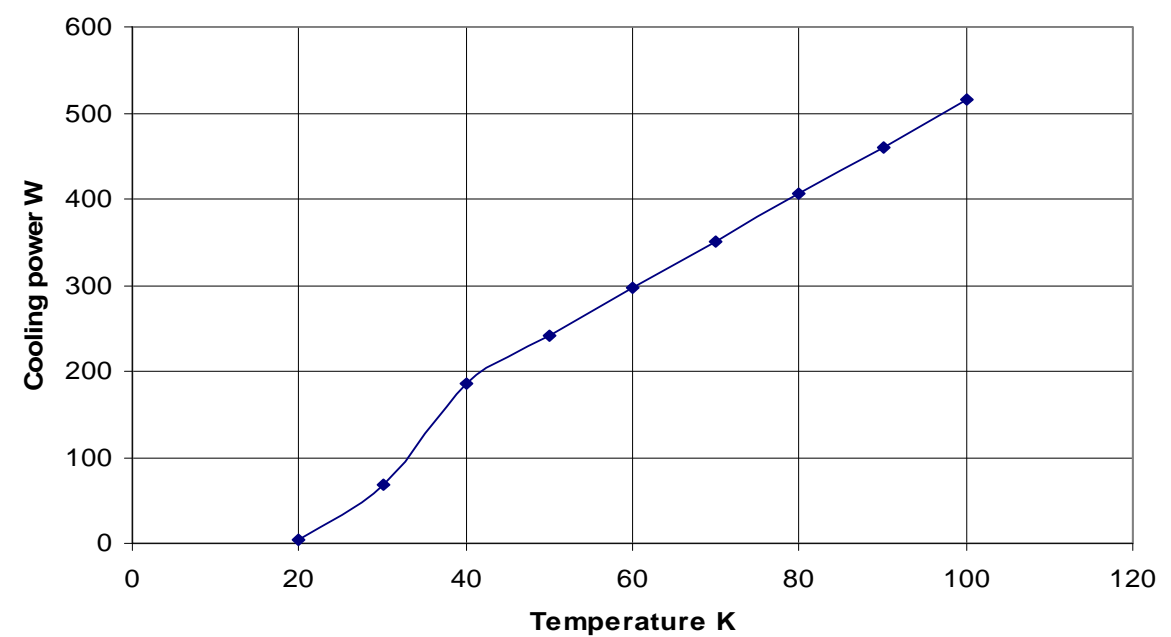

FIGURE 3. Original Sage Model's prediction of cooling power vs temperature at $50 \mathrm{~Hz}$ and 25 bar charge pressure.

Constraints placed on the model were that the CHC200 PWG was to be used as the pressure oscillator; it was the only one available at the time. The displacer was to use the CHC200's 365 mm diameter diaphragms. The PWG was modeled as a very short stroke piston in a cylinder with a surface area and volume that gave a hydraulic diameter in the order of $4 \mathrm{~mm}$. The bounce space was not modeled as it was assumed to provide a constant force to balance the average force on the displacer. A bounce space volume was incorporated in an earlier model and produced a negligible effect on the result but significantly affected the stability of the solver. The displacer and cylinder object in the model is a standard Sage item, which includes a displacer/reciprocator, spring, cylinder wall and displacer shell. The "annulus shuttle/seal/appendix" item from the standard Sage object was removed as the use of diaphragms meant that there was no need for the model to include a clearance gap between the cylinder wall and the displacer. The displacer shell in the model contained the regenerator matrix. The displacer's walls, with their associated temperature gradients and heat leaks were shared between the displacer walls and the cylinder wall objects in the model. Optimization of the regenerator geometry, and intermediate diaphragm area resulted in the performance shown in FIGURE 3. A refrigeration power of $390 \mathrm{~W}$ was predicted at $77 \mathrm{~K}$ with $4.0 \mathrm{~kW}$ of input pressure-volume (PV) power resulting in an indicated Carnot efficiency of 28\%. With a PWG efficiency of $70 \%$ [8], this represents an overall Carnot efficiency of $20 \%$ at $77 \mathrm{~K}$. This performance compares well with other cryocoolers [9] and justified the manufacture of a proof-ofconcept prototype. The amplitude of displacer movement was $0.41 \mathrm{~mm}$, which is much less than the diaphragm's limit of $1.25 \mathrm{~mm}$ indicating that a smaller diaphragm with would be suitable.

\section{PROOF-OF-CONCEPT PROTOTYPE.}

A proof-of-concept prototype was designed and constructed based on parameters from the Sage model. The practicalities of design and manufacture introduced a number of deviations from the model that resulted in reduced performance. The first and most significant was the stiffness of the intermediate diaphragm. The prototype's intermediate diaphragm turned out to be an order of magnitude stiffer than initially modeled. 


\section{Revised Sage prediction of cooling performance}

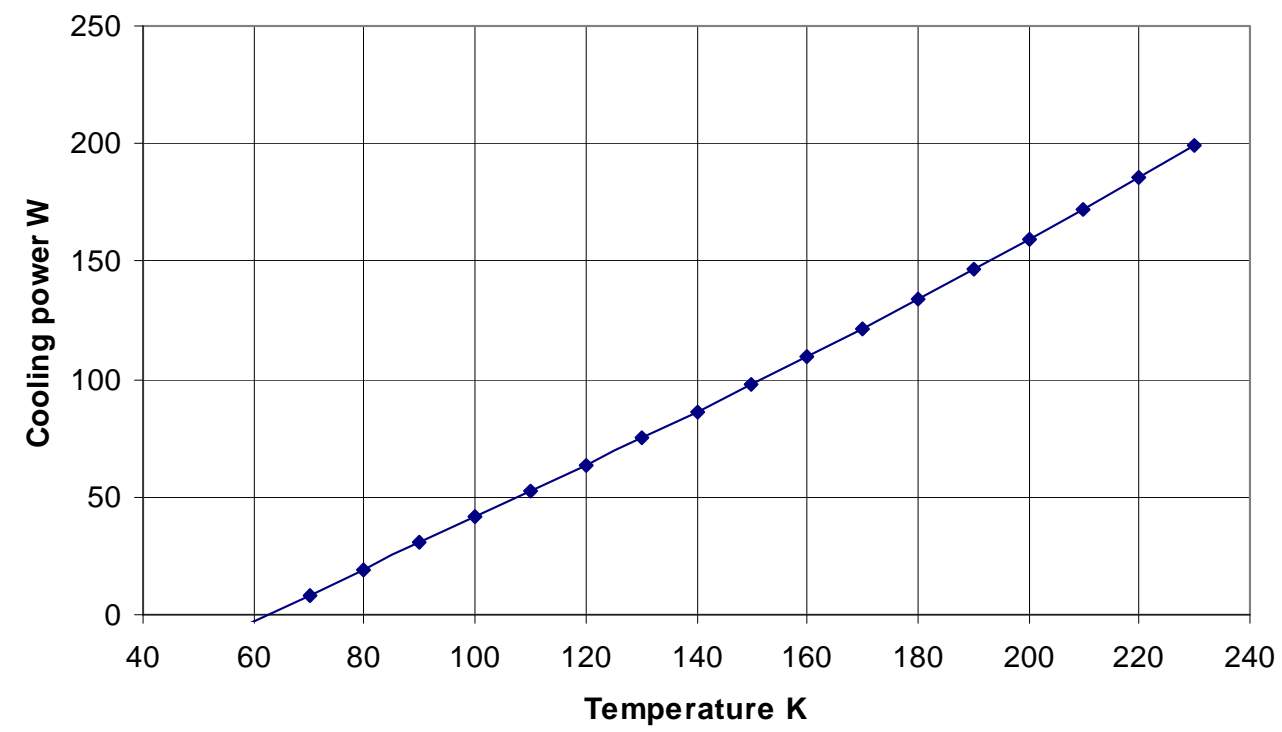

FIGURE 4. Revised model prediction of the as built prototype at $50 \mathrm{~Hz}$ and 25 bar charge pressure.

The stiff diaphragm reduced the displacer movement, resulting in less gas movement through the regenerator. More gas therefore remained on each side of the displacer taking part in compression and expansion without performing any useful function.

The second and almost equally significant deviation was the introduction of dead volumes at the outer radii of the diaphragms. This was a design oversight that resulted in a reduced compression ratio which translated to less input power into the gas and a lower pressure wave for moving the displacer, all reducing cooling power. These modifications were incorporated into the Sage model after construction of the prototype. The revised cooling power prediction is shown in FIGURE 4. Cooling power of $19 \mathrm{~W}$ at $80 \mathrm{~K}$ was predicted with $2.1 \mathrm{~kW}$ of input power. Cryogenic temperatures should still be attainable although $80 \mathrm{~K}$ might be unattainable as parasitic losses not included in the model, such as radiation within the displacer, could exceed the $19 \mathrm{~W}$ of refrigeration available.

\section{EXPERIMENTAL RESULTS}

From its first run the prototype provided noticeable refrigeration. The prototype suffered from an elusive slow leak into the cryostat, limiting the vacuum in the cryostat to an unsatisfactory $1 \mathrm{mbar}$ and resulting in poor insulation throughout tests. The heat load from the cryostat leak was significant, limited the ultimate cold temperature and made cooling power measurements impractical.

A set of experiments was conducted to verify the Sage model's predictions of the prototype's behavior. The verification experiments were conducted without insulation to give access to the displacer position sensor; hence they were conducted around the temperature of $200 \mathrm{~K}$. The charge gas was helium at a pressure of 20 bar. The verification parameters measured were the pressure wave's amplitude and phase, and the displacer movement, amplitude and phase. Phase angles were relative to the pressure wave generator's driving piston.

The driving piston and displacer movements were measured using Keyence LB72 laser transducers, which allowed non-contact direct position measurement. Experiments 
were conducted over a range of frequencies from 20 to $60 \mathrm{~Hz}$. Pressure was measured adjacent to the PWG's diaphragm. Temperature was measured in the centre of the cold plate with a PT100 probe.

Two cases were considered: the first as designed and the second with the intermediate diaphragm removed. The effect of the latter is the spring constant for the displacer was significantly reduced - a positive outcome; the dead volume on the warm side of the displacer was increased by the volume of the bounce space - a negative outcome; and the movement of the displacer was now driven solely by the pressure drop across the regenerator which was not ideal. A modified Sage model was produced to simulate the effect of the second case and predicted reduced cooling power with a no-load temperature of $100 \mathrm{~K}$.

FIGURE 5 shows the pressure wave amplitude and PV powers experimentally measured as compared with the Sage Model. The correlation of the macroscopic parameters of pressure wave amplitude and PV power show that the Sage model provided a reasonable prediction of gas compression and heat transfer inside the machine. Of note was that the slow speed runs approached isothermal compression inside the machine $\left(\mathrm{P}_{\max } / \mathrm{P}_{\min }\right.$ $\left.\sim \mathrm{V}_{\max } / \mathrm{V}_{\min }\right)$ and the high speed runs approached adiabatic behavior $\left(\mathrm{P}_{\max } / \mathrm{P}_{\min } \sim\right.$ $\left.\left(\mathrm{V}_{\max } / \mathrm{V}_{\min }\right)^{1.667}\right)$.

FIGURE 6 shows the displacer movement. The experiment agreed well with the Sage prediction for displacer amplitude for the case with the bounce space. However, with the bounce space removed, the experimental displacer amplitude steadily increased with frequency whilst its prediction was comparatively flat. Sage over predicted the displacer phase angle for the bounce space case, which would lead to poorer cooling of the prototype than predicted. However when the bounce space was removed, the phase angle was larger than predicted, leading to better cooling.

The cool down rate was dominated by the $29.5 \mathrm{~kg}$ stainless steel top on the cold head. An initial fast cooling rate was observed while temperature gradients in the plate became established, and then a long slow descent was observed while it steadily cooled the outer parts of the plate. The lowest temperature recorded for the standard design, with bounce space, was $148 \mathrm{~K}$. This was much poorer than expected.

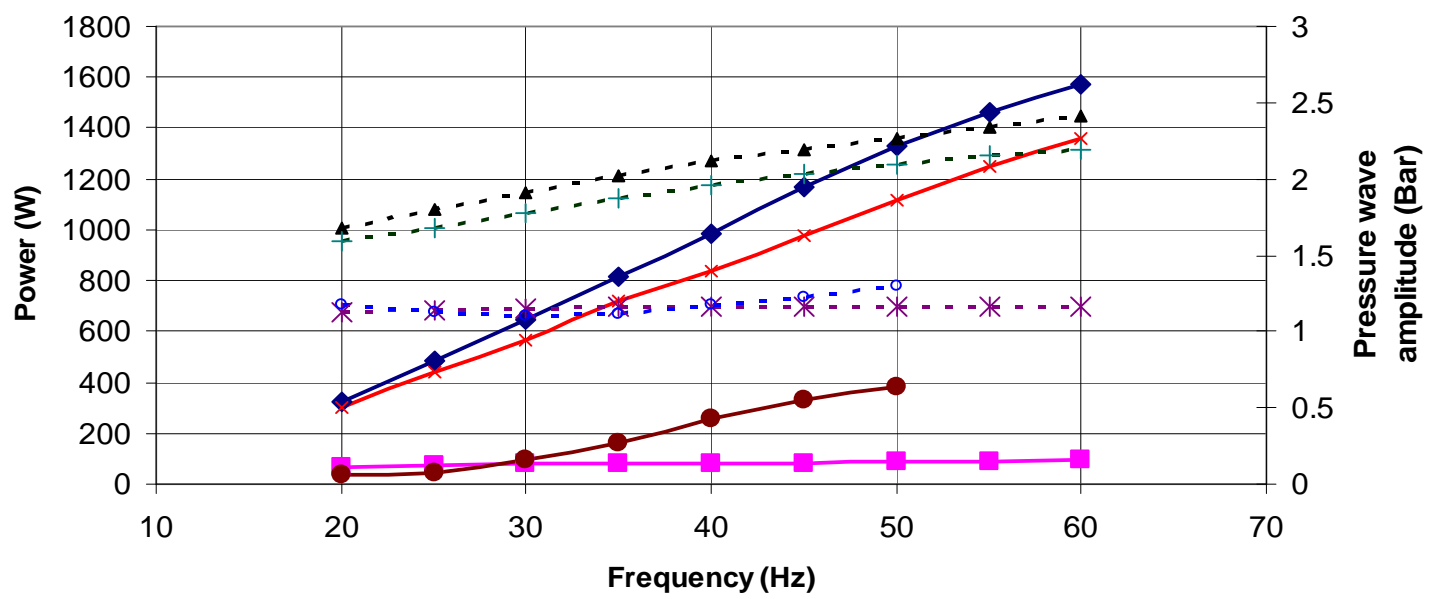

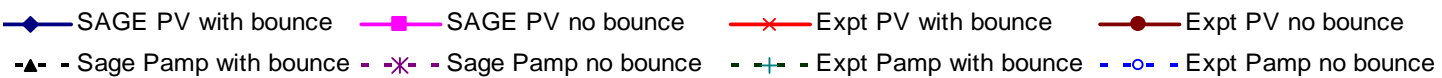

FIGURE 5. Comparison of Sage model and experimental pressure wave amplitude and PV power for first prototype characterization runs. 


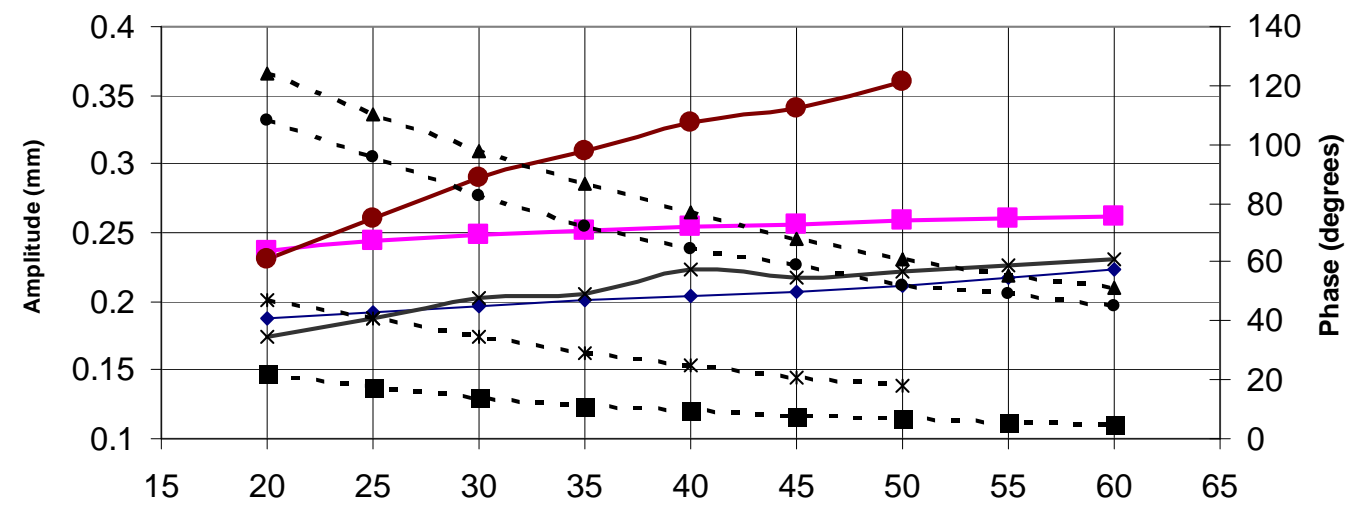

Frequency $(\mathrm{Hz})$

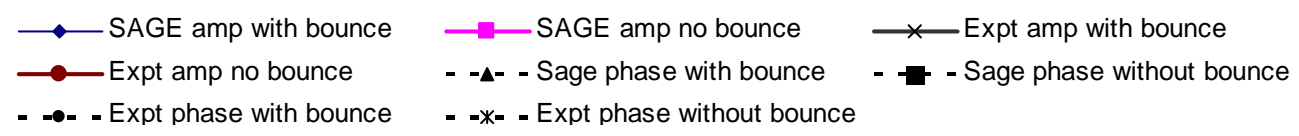

FIGURE 6. Comparison of Sage model and experimental prototype displacer movement for first characterization runs.

Without the bounce space, the prototype achieved a much slower but better cooldown as shown in FIGURE 7. The initial running speed of the machine was $45 \mathrm{~Hz}$, a speed that appeared to work well at higher temperatures, and cooled it to $107 \mathrm{~K}$ in 8 hours. The PWG frequency was then increased to $60 \mathrm{~Hz}$, which cooled it down to $100.5 \mathrm{~K}$ in a further 15 minutes.

\section{DISCUSSION}

The concept of using twin opposed diaphragms to support and seal the displacer of a free piston Stirling cryocooler has been tested and appears feasible according to a Sage model of the system and a proof-of-concept prototype. The coldest temperature achieved by the proof of concept prototype was $100.5 \mathrm{~K}$. This was achieved without the bounce space indicating that the benefits from a softer displacer spring outweighed the loss in displacer phase angle and extra dead volume experienced when the intermediate diaphragm was removed. The Sage model was able to predict the prototype's macroscopic thermodynamic and dynamic behavior, such as the pressure wave magnitude, pressure wave phase, and displacer movement. The Sage predictions held reasonably well with and without the bounce space and intermediate diaphragm. The Sage model indicated a number of areas for improvement of the prototype. A smaller diaphragm for the displacer would be an advantage as it would use more of the diaphragms' potential stroke; reduce the diaphragm area and hence dead volumes; reduce diaphragm area and hence gas forces leading to thinner walls and less conduction losses. IRL have since developed a smaller 60 cc swept volume PWG whose diaphragms can produce the same displacer swept volume as the proof-of-concept machine with a stroke comparable to that of the PWG. The other significant improvement would be the addition of a more traditional 'shaft' to produce a piston-dashpot to achieve the bounce space effect without adding an extra spring in the form of an additional diaphragm. A second prototype design with the above improvements is being manufactured at the time of writing; its Sage model predicts $240 \mathrm{~W}$ of cooling at $77 \mathrm{~K}$ with a Carnot efficiency of $28 \%$. 


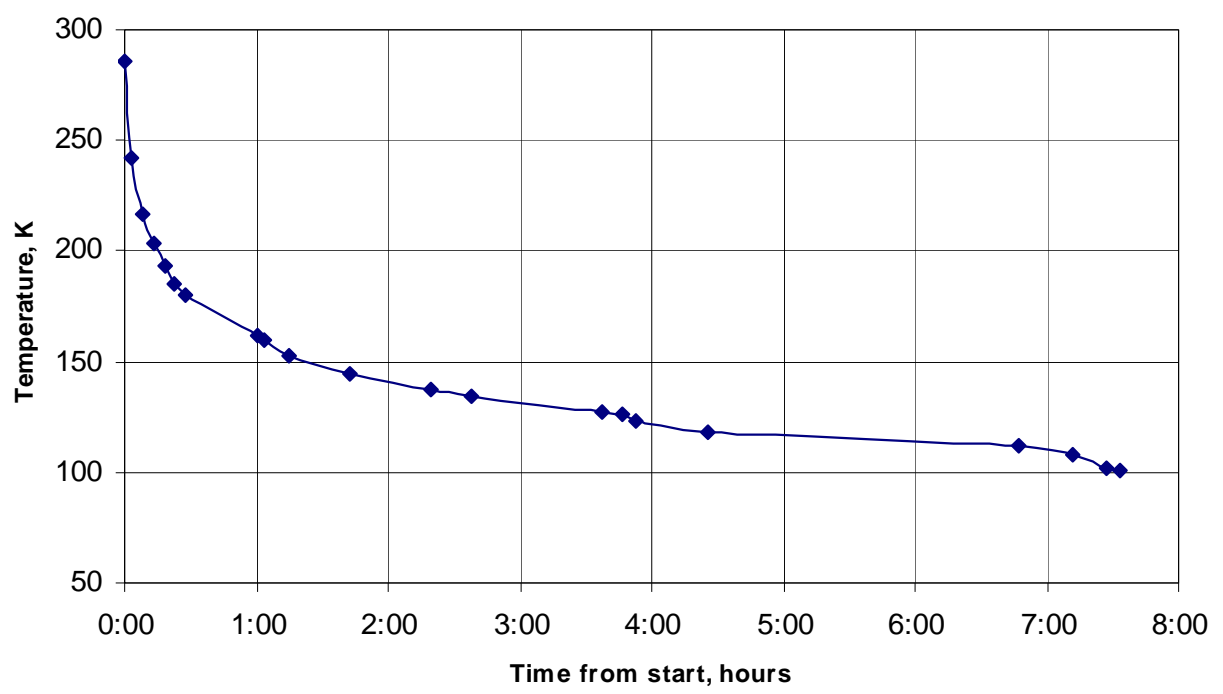

FIGURE 7. Cool down curve of the prototype without bounce space.

\section{ACKNOWLEDGEMENTS}

The authors acknowledge Industrial Research Ltd, New Zealand for support of this work, New Zealand's Ministry for Science and Innovation for funding, the University of Canterbury for supervision of the project and HTS-110 for supporting the commercialization of the technology.

\section{REFERENCES}

1. Caughley A., Haywood D., Wang C., “A Low Cost Pressure Wave Generator Using Diaphragms”, in Advances in Cryogenic Engineering, 53B, AIP. Melville New York, 2008, pp 1122-1129.

2. Beale W. T. "Free Piston Stirling Engines - Some Model Tests and Simulations", in SAE International Automotive Engineering Congress. Detroit Mich. January 1969.

3. Cooke-Yarborough E H, " A 60 Watt thermomechanical generator as the main power source for a major lighthouse" in Proc Xth Int Conf on Lighthouses and other aids to navigation (IALA) Tokyo, Nov 1980

4. About Sunpower web page, www.sunpower.com

5. Cooke-Yarborough E H, “A Striling-cycle generator powered by radio-isotope”, in $8^{\text {th }}$ International Stirling Engine Conference and Exhibition, May 1999, Ancona. ISEC97051.

6. Walker G, Bingham E R, "Stirling Cryocoolers”, in Low Capacity Cryogenic Refrigeration, Edited by R Scurlock, Clarendon Press, Oxford, 1994, pp. 123-125.

7. Gedeon, D. Sage Home Page, www.sageofathens.com

8. Caughley A., Wang C., "Development of a Diaphragm Pressure Wave Generator for Cryocoolers", in Cryocoolers 15,edited by S D Miller, ICC Press, Boulder Colorado, 2009, pp 309-315.

9. Tranchon J et al, "Design of a very large pulse tube cryocooler for HTS cable application" in Advances in Cryogenic Engineering 51A edited by J Weisend et al, AIP Melville New York, 2006, pp 661-668 